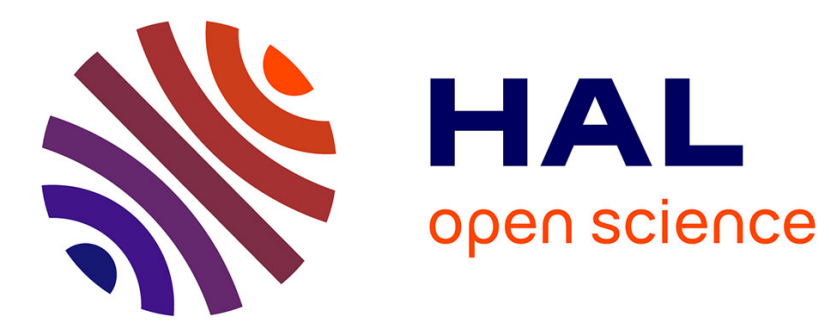

\title{
Cooperative luminescence in an ytterbium-doped silica fibre
}

Sylvain Magne, Youcef Ouerdane, Michel Druetta, Jean-Pierre Goure, Pierre Ferdinand, Gérard Monnom

\section{- To cite this version:}

Sylvain Magne, Youcef Ouerdane, Michel Druetta, Jean-Pierre Goure, Pierre Ferdinand, et al.. Cooperative luminescence in an ytterbium-doped silica fibre. Optics Communications, 1994, 111 (3-4), pp.310-316. hal-00469779

\section{HAL Id: hal-00469779 https://hal.science/hal-00469779}

Submitted on 6 Apr 2010

HAL is a multi-disciplinary open access archive for the deposit and dissemination of scientific research documents, whether they are published or not. The documents may come from teaching and research institutions in France or abroad, or from public or private research centers.
L'archive ouverte pluridisciplinaire HAL, est destinée au dépôt et à la diffusion de documents scientifiques de niveau recherche, publiés ou non, émanant des établissements d'enseignement et de recherche français ou étrangers, des laboratoires publics ou privés. 


\title{
Cooperative luminescence in an ytterbium-doped silica fibre
}

\author{
S. Magne ${ }^{a}$, Y. Ouerdane ${ }^{b}$, M. Druetta ${ }^{b}$, J.P. Goure ${ }^{b}$, P. Ferdinand a ${ }^{a}$ G. Monnom ${ }^{c}$ \\ * LETI (CEA - Technologies Avancées), DEIN/SPE - Centre d'Etudes de Saclay, F-91191 Gif-sur-Yvette, France \\ b URA CNRS 842 - Laboratoire Traitement du Signal et Instrumentation, \\ 23, rue du Dr Paul Michelon, F 42023 St Etienne, France \\ - URA CNRS 190 - Laboratoire de Physique de la Matière Condensée, \\ Parc Valrose, F 06108 Nice, France
}

Received 6 October 1993; revised manuscript received 6 May 1994

\begin{abstract}
We report the first spectral and power-law modeling of cooperative luminescence in an ytterbium-doped optical silica fibre structure enabling the creation of new saturation effects. We believe that the low-concentration transfer originates in the ytterbium-oxide molecule itself and is consequently due to the inherently oxidizing MCVD process.
\end{abstract}

\section{Introduction}

Cooperative luminescence, first observed in 1970 by Nakazawa and Shionoya [1], is one of four cooperative optical phenomena (cooperative absorption and luminescence, combination absorption and luminescence) observed either in crystals [1,2] or in glasses [3]. These effects are responsible for the quenching of the luminescence of dopants in glass so that their concentrations must be kept low [4].

We report here the first modeling, to our knowledge, of this effect in an optical fibre.

By contrast to bulk materials, the propagation of light in fibres is no longer diffraction-limited, so that a very small spot size can be maintained over very long interaction lengths. Therefore, the intensity of the light confined in the core of the fibre can easily reach several $\mathrm{MW} \mathrm{cm}^{-2}$ in continuous wave when carrying powers of only some $\mathrm{mW}$. So, nonlinear phenomena are far easier to observe in fibres than in bulk materials. The $\mathrm{Yb}^{3+}$ ion has been widely studied $[1-3]$ since no transition occurs in the visible range. The visible luminescence is thus clearly correlated to a cooperative phenomenon.

\section{Experimental}

The $\mathrm{GeO}_{2}-\mathrm{P}_{2} \mathrm{O}_{5}$-codoped fibre preform is fabricated using the Modified Chemical Vapor Deposition (MCVD) technique. The ytterbium oxides are incorporated into the core by the solution-doping method [5]. The preform was then drawn into a monomode fibre of $4 \mu \mathrm{m}$ core diameter. We measured the fluorescence lifetime of the ${ }^{2} F_{5 / 2}$ excited level (Fig. 1) by acousto-optic modulation and it yielded $\tau \approx 0.97 \mathrm{~ms}$. We inferred that our fibre was free of aggregates since only one decay component was observed, as experimentally described in previous experiments [4]. We measured the $\mathrm{Yb}_{2} \mathrm{O}_{3}$ concentration by neutron activation analysis (NAA) [6] and it yielded $85 \mathrm{ppm}$ molar. The fibre was excited by an argon-ion laser-pumped-titanium-sapphire laser and the spectra were recorded using a Czerny-Turner 


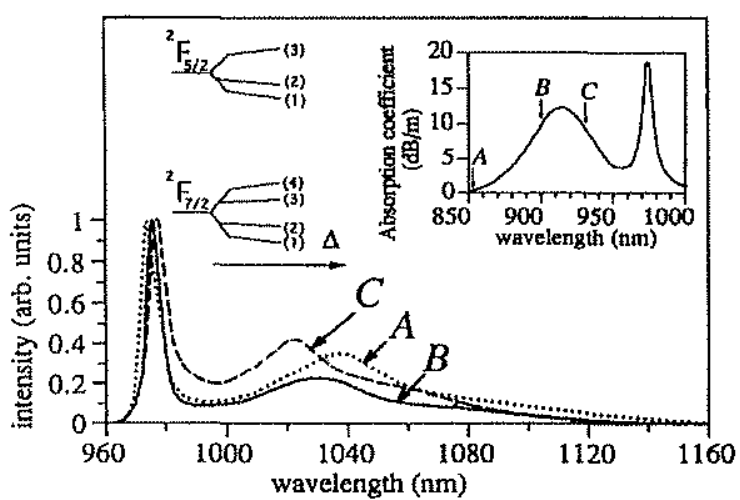

Fig. 1. Site-selective room-temperature fluorescence spectra (normalized to unity) of the monomode $\mathrm{Yb}^{3+}$-doped fibre upon several excitation wavelengths. Insert shows the room-temperature fibre absorption spectrum and the energy-level fluorescenceline-narrowing symbolic diagram relative to site distribution $\Delta$.

spectrometer equipped with a photomultiplier.

We observed a green luminescence, transversely visible by the naked eye, when the fibre was excited within the absorption band of the $\mathrm{Yb}^{3+}$ ion (Fig. 1). We estimated the cooperative luminescence power to be six orders of magnitude lower than the infra-red single-ion fluorescence power. This result is of the same order than that previously reported [1]. The cooperative luminescence spectra do not change with temperature $(77 \mathrm{~K}$ to room temperature) owing to the very large inhomogeneous broadening in glasses. Some little changes appear in the spectra when varying the fibre length (Fig. 2). The cooperative luminescence power evolves quadratically for excitation powers lower than the saturation power in this fibre. Then it evolves linearly as the excitation power exceeds this saturation power to eventually saturate at very high excitation powers (Fig. 3 ) even though only a small portion of fibre corresponding to the injected-part of the fibre is actually generating cooperative luminescence.

\section{Discussion}

Fluorescence studies reveal that the ${ }^{2} \mathrm{~F}_{5 / 2} \rightarrow{ }^{2} \mathrm{~F}_{7 / 2}$ transition is very inhomogeneously broadened on account of the high disorder of the configuration environment of the $\mathrm{Yb}^{3+}$ ions. Site-selective fluorescence spectra are represented in figure 1 and are indeed very
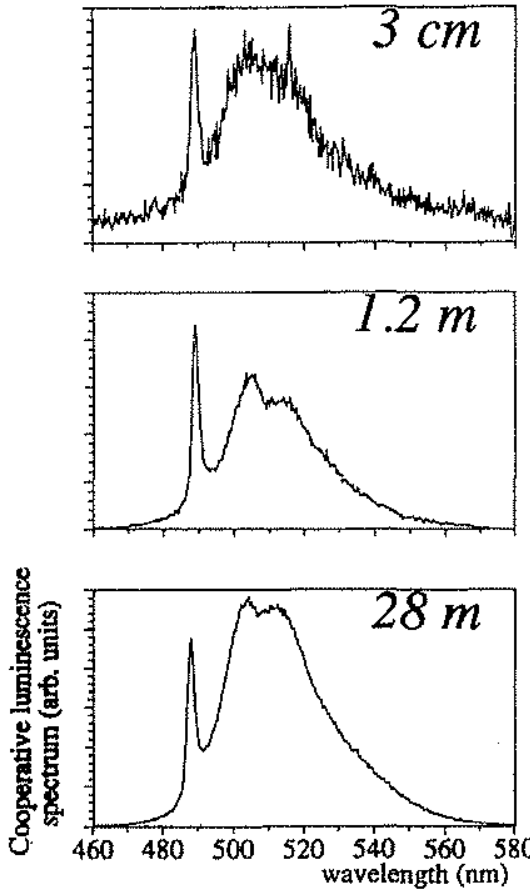

Fig. 2. Evolution of the spectral shape of the cooperative luminescence spectra for several fibre lengths (excitation wavelength $900 \mathrm{~nm}$, excitation power $60 \mathrm{~mW}$ ).

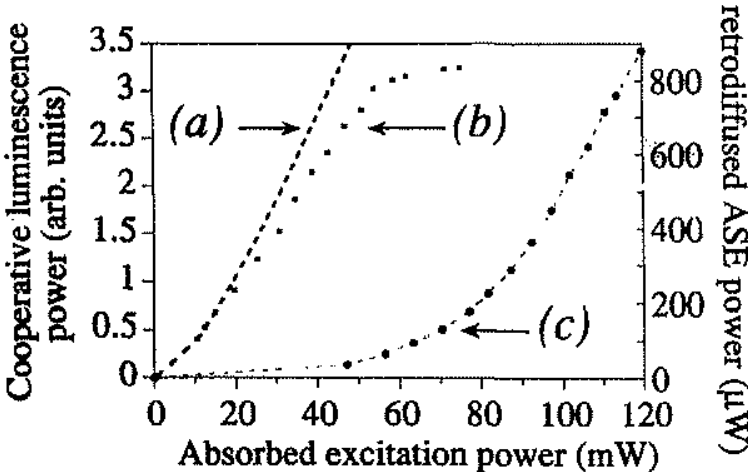

Fig. 3. Evolution of the cooperative luminescence power [(a) theory neglecting the ASE saturation (Eq. 11), (b) experiment] and the counter-propagating ASE power (c) with respect to $a b-$ sorbed excitation power (excitation wavelength $900 \mathrm{~nm}$, the fibre is $28 \mathrm{mlong}$ ).

pump-wavelength dependent. This disorder is also responsible for fluctuations in the short-range $\mathrm{Yb}^{3+}-\mathrm{Yb}^{3+}$ interaction [2]. $\mathrm{f}-\mathrm{f}$ transitions of rareearth ions occupying sites lacking inversion symmetry are predominantly interpreted as forced-electric- 
dipole in nature because of the $4 \mathrm{f}^{n-1} 5 \mathrm{~d}$ electronic states admixture lifting the parity forbiddeness of the electric dipole f-f transition (Laporte's rule).

We propose both a spectral and power-law modeling of the cooperative luminescence transfer in a fibre structure.

\subsection{Spectral modeling}

Since the rare-earth ions are characterized by a weak phonon coupling, we can accurately assume a very low pair interaction involving only the singleion energy-level diagram.

Furthermore, assuming no correlation between the pair interaction and the configuration environment of the $\mathrm{Yb}^{3+}$ ions, the cooperative spectrum $A(\nu)$ can be described as the self-convolution of the single-ion fluorescence spectrum $\Lambda_{e}(\nu)$ [2] (Fig. 4) provided that the cooperative transfer rate, at a given frequency $\nu$, results in the summation of every infra-red contributions, that is

$\nu_{\text {donor }}+\nu_{\text {acceptor }}=\nu$,

where $\nu$ is the frequency of the cooperative luminescence photon and $\nu_{\text {donor }}$ and $\nu_{\text {acceptor }}$ are the infra-red transition frequencies of the donor and acceptor ion respectively. The cooperative transfer rate can therefore be written as

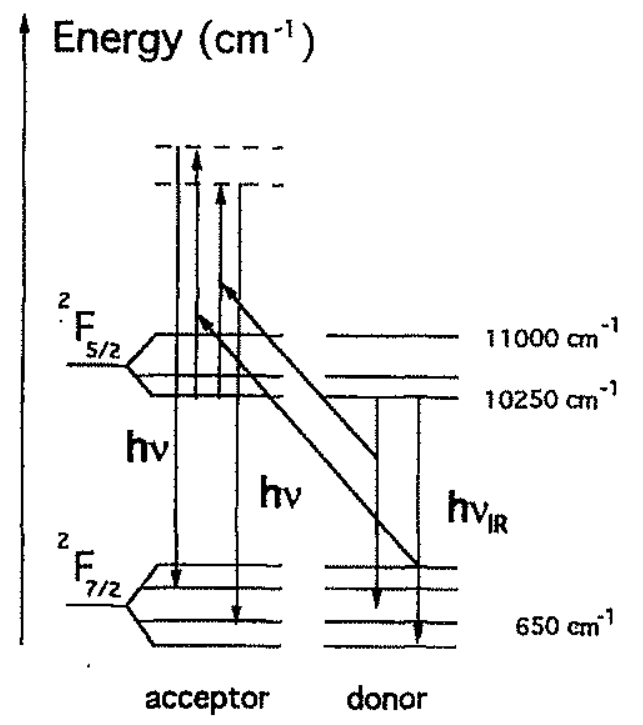

Fig. 4. Cooperative tuminescence process schematic diagram.
$\Lambda(\nu)=\int_{\substack{\text { emission } \\ \text { spectrum }}} \Lambda_{\mathrm{e}}\left(\nu_{\text {donor }}\right) \Lambda_{\mathrm{c}}\left(\nu_{\text {acceptor }}\right) \mathrm{d} \nu_{\text {donor }}$

Therefore, taking into account the summation condition (1), the cooperative luminescence spectrum can be described as a convolution of the infrared fluorescence spectrum by itself:

$\Lambda(\nu)=\Lambda_{\mathrm{e}} * A_{\mathrm{e}}\left(\nu_{\text {donor }}\right)$

Fig. 5 depicts the comparison between the experimental site-selective cooperative luminescence spectra and the calculated self-convoluted infra-red fluorescence spectra for three different excitation wavelengths and a fibre length of $1.2 \mathrm{~m}$. The cooperative luminescence spectrum shows three peaks corresponding to three different occurrences of the infra-red fluorescence spectrum. A first peak centered
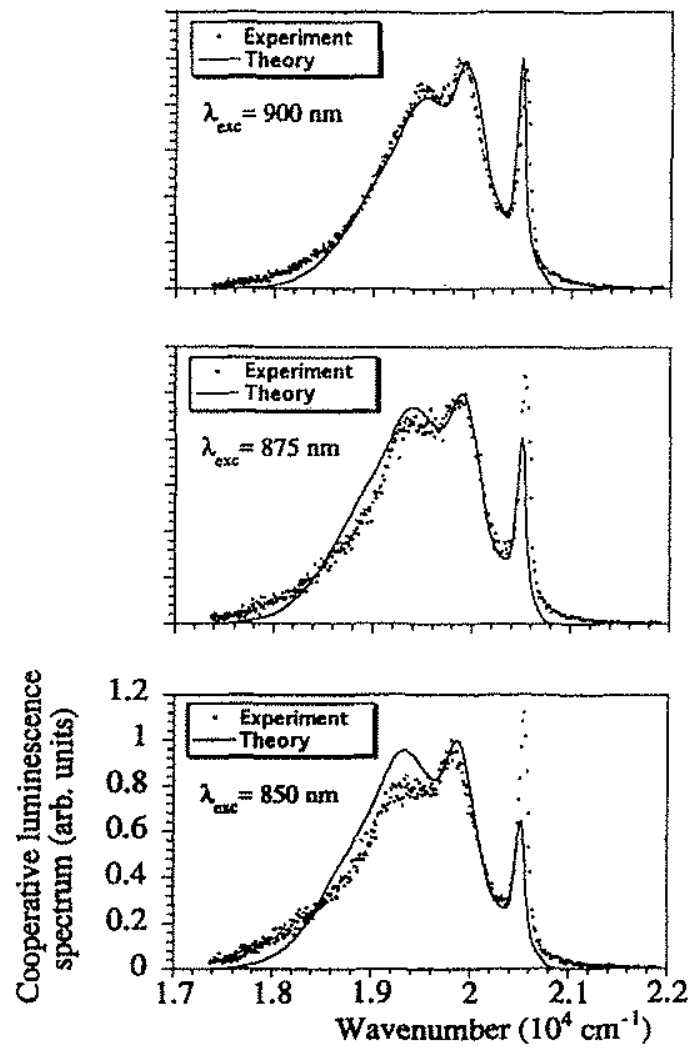

Fig. 5. Comparison between experimental and calculated site-selective cooperative luminescence spectra. 
around $20490 \mathrm{~cm}^{-1}$ corresponds to the occurrence of the two thin fluorescence peaks centered at $976 \mathrm{~nm}$. A second peak, around $19900 \mathrm{~cm}^{-1}$, corresponds to the occurrence of the thin fluorescence peak centered at $976 \mathrm{~nm}$ and the broad fluorescence peak centered at $1025 \mathrm{~nm}$. A third peak, around $19530 \mathrm{~cm}^{-1}$, corresponds to the occurrence of the two broad peaks centered at $1025 \mathrm{~nm}$. The thin fluorescence peak centered at $976 \mathrm{~nm}$ corresponds to a transition ending on the ground-state which subsequently suffers reabsorption losses. Therefore, the infra-red fluorescence spectrum is modified in the way that this peak is lower in intensity than expected and this modification accounts for the discrepancies observed between the experimental and calculated cooperative luminescence spectra especially at lower wavelengths ( 875 and $850 \mathrm{~nm}$ ). The comparison is very good when the excitation takes place at the top of the absorption band where the pump saturation power is very low (around $3 \mathrm{~mW}$ at $900 \mathrm{~nm}$ in this fibre). When exciting the top of the absorption band, a very high population inversion can be achieved leading to very small ground-state reabsorption losses and yielding an accurate spectral modeling.

\subsection{Power-law modeling}

As described elsewhere [7], we define $N_{2}$ as the population of the Stark sub-level (1) of the upper level ${ }^{2} \mathrm{~F}_{5 / 2}$ and $N_{1}$ as the population of the lower level ${ }^{2} \mathrm{~F}_{7 / 2}$. These populations can be written, in absence of Amplified Spontaneous Emission (ASE) saturation, as

$N_{1}=\frac{1}{1+\beta} N, \quad N_{2}=\frac{\beta}{1+\beta} N, \quad N_{3}=0$,

where the population $N_{3}$ of the excited Stark sub-levels (2) and (3) of the ${ }^{2} \mathrm{~F}_{5 / 2}$ manifold (Fig. 1) is assumed to be negligible. $N$ is the concentration of $\mathrm{Yb}^{3+}$ ions and $\beta$ is the ratio of the injected intensity to the saturation intensity defined as $I_{\text {sat }}=h \nu_{\mathrm{p}} / \sigma_{\mathrm{p}} \tau$, where $\sigma_{\mathrm{p}}$ is the pump transition interaction cross section at the pump frequency $\nu_{p}$ and $\tau$ is the ${ }^{2} \mathrm{~F}_{5 / 2}$ excited level lifetime. Assuming a transfer rate evolving as $N_{2}^{2}$, since it is a two-photon process, the modeling requires integrating over the monomode profile and over the interaction length taking into account the longitudinal evolution of the inversion. The guided monomode profile can be well approximated by a gaussian profile [7] and the modeling yields the following transfer rate:

$\frac{N_{\mathrm{up}}}{N}=\int_{0}^{L} \int_{0}^{a^{2} / W_{b}^{2}} \frac{\beta^{2} p(z)^{2} \exp (-2 x)}{(1+\beta p(z) \exp (-x))^{2}} \mathrm{~d} x \mathrm{~d} z$,

where $x=r^{2} / W_{\mathrm{p}}^{2}$, and $r$ and $z$ are respectively the radius and abscissa coordinates of the fibre.

Since the whole core is codoped with $\mathrm{Yb}_{2} \mathrm{O}_{3}$, the integration is carried up until the fibre core radius $a$. $W_{\mathrm{p}}$ is the half-width at half-maximum guided-mode profile and $p(z)$ represents the longitudinal pump evolution: $p(z)=P_{\mathrm{p}}(z) / P_{\mathrm{p}}(0)$, where $P_{\mathrm{p}}(z)$ is the residual pump power at a length $z$ and $P_{\mathrm{p}}(0)=P_{\text {inj }}$ is the injected pump power.

The cooperative luminescence power is not reab. sorbed since no transition occurs in the visible range and the distributed losses are absolutely negligible for fibres a few meters long.

The longitudinal evolution of the pump power obeys the pumping equation $[6,7]$ :

$\frac{\mathrm{d} p(z)}{p(z)}=-\sigma_{p} N_{\mathrm{l}}(z) \mathrm{d} z$.

This pumping equation is valid since the pump transition is homogeneously broadened at the top of the ${ }^{2} \mathrm{~F}_{5 / 2}$ Stark manifold where the excitation takes place. Solving Eq. (6) leads to an analytical form for $\mathrm{d} p(z) /$ $\mathrm{d} z$.

$\frac{\mathrm{d} p(z)}{\mathrm{d} z}=\frac{\alpha_{\mathrm{p}}}{\beta} \ln \left(\frac{1+\beta p(z)\left(1-\eta_{\mathrm{p}}\right)}{1+\beta p(z)}\right)$,

which, inserted into (5), leads to a formulation of the cooperative transfer rate:

$$
\begin{aligned}
N_{\mathrm{up}} & =\frac{P_{\mathrm{abs}}}{\left(h \nu_{\mathrm{p}} / \tau\right)}-\frac{P_{\mathrm{inj}}}{\left(h \nu_{\mathrm{p}} / \tau\right)} \eta_{p} \\
& \times \int_{1}^{p} \frac{\beta p \mathrm{~d} p}{(1+\beta p)\left[1+\beta p\left(1-\eta_{\mathrm{p}}\right)\right]} \\
& \times\left[\ln \left(\frac{1+\beta p\left(1-\eta_{\mathrm{p}}\right)}{1+\beta p}\right)\right]^{-1}
\end{aligned}
$$

where $P_{\mathrm{abs}}=\pi W_{\mathrm{p}}^{2} I_{\mathrm{abs}}$ is the absorbed excitation power, $P_{\mathrm{inj}}$ is the injected power and $\eta_{\mathrm{p}}$ is an overlap coefficient of the guided mode interacting with the doped core defined as [7] 
$\eta_{\mathrm{p}}=\frac{2}{W_{\mathrm{p}}^{2}} \int_{0}^{a} \Psi_{\mathrm{p}}(r) r \mathrm{~d} r$

where $\Psi_{p}(r)$ is the gaussian guided-mode profile (normalized to its maximum).

For the fibre used in this experiment, the saturation power $P_{\text {sat }}=\pi W_{\mathrm{g}}^{2} I_{\text {sat }}$ was around $2.8 \mathrm{~mW}$ at 900 $\mathrm{nm}$ and $8 \mathrm{~mW}$ at $875 \mathrm{~nm}$ and the overlap coefficient was 0.6 .

Eq. (7) was solved numerically by the fourth-order Runge-Kutta method and yielded the value of $p_{\mathrm{L}}$ (i.e. the fraction of power exiting the fibre of length $L$ ) versus $P_{\text {inj. }}$. This numerical procedure enabled us to determine iteratively the values of $P_{\text {inj }}$ and $p_{\mathrm{L}}$ versus the residual power exiting the fibre $\left(P_{\text {inj }} p_{\mathrm{L}}\right)$, this latter power being measured. The absorbed excitation power $P_{\text {abs }}$ is subsequently deduced via the simple relation:

$P_{\mathrm{aba}}=P_{\text {inj }}\left(1-p_{\mathrm{L}}\right)$.

Considering a very long length of fibre as compared to the absorption length $L_{\mathrm{B}}=1 / \alpha_{\mathrm{p}}$ (typically, around $30 \mathrm{~cm}$ at the top of the absorption band in this fibre) the power law (8) can be simplified using a simple first-order series expansion as $p_{\mathrm{L}}$ being close to zero. All the excitation power is therefore absorbed, so that $P_{\mathrm{abs}} \approx P_{\mathrm{inj}}$ and $\mathrm{Eq} .(8)$ reduced to

$\underset{L \rightarrow L_{\mathrm{B}}}{N_{\mathrm{up}}}=\frac{P_{\mathrm{abs}}}{\left(h \nu_{\mathrm{p}} / \tau\right)}\left(1-\frac{\ln \left(1+\beta\left(1-\eta_{\mathrm{p}}\right)\right)}{\left(1-\eta_{\mathrm{p}}\right) \beta}\right)$.

Using a first-order series expansion since $\beta$ is close to zero, Eq. (11) evolves quadratically as a classical twophoton process for very low powers compared to the saturation power:

$\underset{\substack{l \rightarrow L_{\mathrm{B}} \\ \beta \rightarrow 0}}{N_{\mathrm{up}}} \cong \frac{P_{\mathrm{abs}}^{2}}{\left(h \nu_{\mathrm{p}} / \tau\right)} \frac{1}{2 P_{\mathrm{sat}}}\left(1-\eta_{\mathrm{p}}\right)$

Conversely, considering a very short length of fibre as compared to the absorption length, one can approximate $p_{\mathrm{L}}$ to be very close to unity in the denominator of equation (8), so that it is reduced to:

$$
\begin{aligned}
& \underset{c \ll L_{\mathrm{B}}}{N_{\mathrm{up}}}=\frac{P_{\mathrm{abs}}}{\left(h \nu_{\mathrm{p}} / \tau\right)} \\
& \quad \times\left\{1+\frac{\beta \eta_{\mathrm{p}}}{(1+\beta)\left[1+\beta\left(1-\eta_{\mathrm{p}}\right)\right]}\right.
\end{aligned}
$$

$$
\left.\times\left[\ln \left(\frac{1+\beta\left(1-\eta_{p}\right)}{1+\beta}\right)\right]^{-1}\right\} .
$$

Once again, using a first-order series expansion in $\beta$, Eq. (13) evolves quasi-quadratically for low excita tion powers:

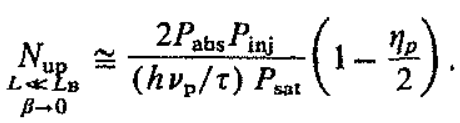

This power-law modeling is valid based on two major assumptions:

Firstly, it assumes a pumping yield close to unity. This condition is seldom met in practice because of the residual thermal population (obeying Boltzmann statistics) of the higher levels of the upper Stark manifold. So, the inversion is limited by the stimulated emission on the pump transition. This "backpumping" [8] is proportional to the thermal population of the pumped Stark level and decreases rapidly as the energy shift between the pump transition energy and the lower-level Stark manifold energy increases. This condition is verified for pumping wavelengths lower than $900 \mathrm{~nm}$ for ytterbium ions at room temperature [6].

Secondly, this model assumes the absence of Amplified Spontaneous Emission (ASE) saturation. This condition is not met for high-length-fibres (typically several absorption lengths-long) submitted to high excitation powers. The complete model including ASE saturation is very lengthy and tedious so that it is desirable to work with short fibre lengths. The ASE saturation accounts for the linear and saturation regime occurring at high excitation powers on high lengths.

The ASE power evolution was measured by inserting a dichroic mirror, which totally reflects the counter-propagating ASE power, while transmitting most of the pumping power through. At the end of 28 $m$ of fibre, the transmitted ASE power was of the order of some $\mathrm{nW}$, while the counter-propagating ASE power reached several $\mathrm{mW}$ and therefore exceeded the saturation power (estimated to be around 700 $\mu \mathrm{W})$.

Fig. 3 shows that the cooperative luminescence power started to saturate as the ASE power began to grow (the threshold of the single-pass counter-propagating ASE is around $60 \mathrm{~mW}$ in absorbed pump power at an excitation wavelength of $900 \mathrm{~nm}$ ). This 
effect explains why the cooperative luminescence power saturated even though a very small portion of the fibre was actually excited and generated cooperative luminescence: any additional excitation power was directly converted into ASE power and the population inversion was "clamped" to the ASE threshold inversion.

Reabsorption losses were also wavelength-dependent so that a transition corresponding to a three-level scheme (i.e., a transition terminating on the groundstate) suffered much more reabsorption losses than a four-level one (terminating on inner less-populated levels). The longer the transition wavelength, the smaller the thermal population of the terminating level and the consequent reabsorption will be. This accounts for the slight changes of shape of the cooperative luminescence spectra as the fibre length increases. We indeed observe that the short-wavelength-part of the spectra was more reabsorbed than the longer-wavelength-part (Fig. 2). Finally, Fig. 6 shows the comparison between the experimental power law and the fit with the Eq. (13) for two different excitation wavelengths. The absorbed excitation power $P_{\text {abs }}$ is obtained versus $P_{\text {inj }}$ according to the calculation procedure previously described. The two series correspond to a same set of measurements and the fit is quite good because of the absence of saturating ASE on so short a length of fibre $(3 \mathrm{~cm})$.

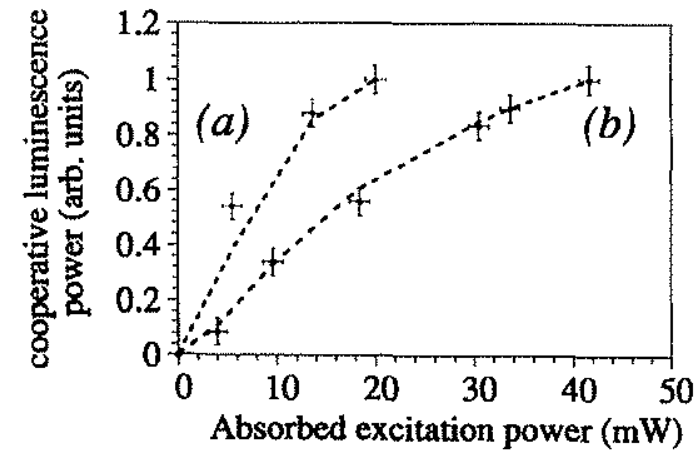

Fig. 6. Evolution of the cooperative luminescence power as a function of the absorbed excitation power for two excitation wavelengths: (a) $900 \mathrm{~nm}$, (b) $875 \mathrm{~nm}$. A short length of fibre (3 $\mathrm{cm}$ ) was spliced to clear fibres in order to observe the fluorescence.

\section{Conclusion}

We show that cooperative luminescence is still present in phosphorous-codoped silica glass fibres even at a very low concentration of $\mathrm{Yb}_{2} \mathrm{O}_{3}(85 \mathrm{ppm}$ molar). We proposed a simple spectral modeling taking into account the site-selective excitation and involving only the single-ion energy-level diagram. This model is very accurate, proving the validity of the convolution model in glass and the absence of correlation between fluctuations in pair interaction and fluctuations in the configuration environment of the $\mathrm{Yb}^{3+}$ ions [2]. The discrepancies observed between experimental and calculated site-selective cooperative luminescence spectra were due to ground-state reabsorptions owing to the transition corresponding to a pure three-level scheme at $976 \mathrm{~nm}$. The fibre structure enabled confining a huge power density over very long interaction lengths. We then observed saturation effects, never observed before in bulk materials, since three-level population inversion is very hard to achieve in free-space upon continuous-wave excitation. As cooperative processes are increased in fibre structure, the Amplified Spontaneous Emission saturation was magnified to the same amount and plagued the cooperative luminescence power growth. This experiment should therefore preferably be performed on small lengths in order to avoid these saturation effects, while still achieving a very high transfer rate due to the optical confinement.

By comparison with other experiments performed on highly ytterbium-doped ZBLAN fibres [9] which have shown little if not cooperative luminescence, we infer that this cooperative energy transfer originates in the oxide molecule itself. As it is well known, silica is a matrix of poor spectroscopic and doping properties inducing clustering [4]. Clustering is very harmful since it degrades considerably the quantum efficiency of fibre lasers and fibre amplifiers so that the estimation of this cooperative transfer rate is of great importance for the improvement of such devices. This experiment proves that transfer phenomena, inducing the quenching of the fluorescence, still occur even in the absence of aggregates. According to Auzel [9], we consider that clustering appears since two ions exchange energy in a cooperative way. Cooperative luminescence thus shows evidence of the amount of clustering. 
Therefore, this simple experiment provides a very useful quantitative and qualitative way to characterize different host matrices by using the ytterbium ion as a probe.

Further information can be inferred by performing a time-resolved site-selective experiment. This would allows us to determine quantitatively the transfer rate and the origin of this transfer.

\section{Acknowledgements}

S. Magne wants to thank B. Jacquier of Laboratoire de Physico-Chimie des Matériaux Luminescents (LPCML URA CNRS 442) for fruitful discussions and Commissariat à l'Energie Atomique (CEALETI-DEIN) for financial support and permission to publish this paper.

\section{References}

[1] E. Nakazawa and S. Shionoya, Phys. Rev. Lett. 25 (1970) 1710.

[2] V.V. Ovsyankin, in: Spectroscopy of solids containing rareearth ions, eds, A.A. Kaplyanskii and R.M. Mc Farlane (North Holland, Amsterdam, 1987) p. 344.

[3] A.V. Dmitryuk, G.O. Karapetyan, V.I. Kosyakov, B.M. Makushkin and V.A. Shirokshin, Opt. Spectrosc. 37 (1974) 335.

[4] B.J. Ainslie, S.P. Craig-Ryan, S.T. Davey, J.R. Armitage, C.G. Atkins, J.F. Massicott and R. Wyatt, IEE Proc. J 137 (1990) 205.

[5] J.E. Townsend, S.B. Poole and D.N. Payne, Electron. Lett. 23 (1987) 329.

[6] S. Magne, PhD Thesis, Université de Saint-Etienne (1993).

[7] M.J.F. Digonnet, SPIE 1171 (1989) 8.

[8] A. Siegman, Lasers (University Science Books, Mill Valley, USA, 1986)

[9] F. Auzel, D. Meichenin, F. Pellé and P. Goldner, Optical Materials, to be published. 